\title{
Effects of social condition and estrous cycle on time-budgeting practices of female hamsters
}

\author{
KENNETH J. FORAND \\ State University of New York at New Paltz, New Paltz, New York \\ and \\ DANIEL Q. ESTEP \\ University of Georgia, Athens, Georgia
}

\begin{abstract}
The behavior of six adult female Syrian golden hamsters (Mesocricetus auratus) was observed across the estrous cycle and under three different social conditions: female alone, castrated male present, and intact male present. When alone, females spent most of their time in bodily maintenance activities such as grooming, eating, and sleeping. Behavior did not vary across the estrous cycle and was remarkably similar to that shown by males housed under similar conditions. In the presence of either castrated or intact males, females spent more time engaged in exploration and less time in locomotor and restless movements than when alone. Sexual behavior and food hoarding were influenced by both social condition and stage of the estrous cycle. We conclude that some behavior patterns of females are influenced by social environment alone, and others are influenced by both social conditions and reproductive conditions.
\end{abstract}

Gonadal hormones have been shown to have a variety of organizational and activational influences on mammalian behavior. Beatty (1979) and Leshner (1979) provided extensive reviews of the literature concerning the effects of gonadal hormones on reproductive and nonreproductive behavior (e.g., locomotor activity, aggression, and food intake) of rodents and primates.

Investigators of hormone/behavior relationships have found that a number of behavior patterns fluctuate systematically across the estrous cycles of female hamsters. Affected behavior patterns include gnawing (Morin, 1978), flank marking and vaginal marking (Johnston, 1977), feeding (Morin \& Fleming, 1978), food hoarding (Estep, Lanier, \& Dewsbury, 1978), and emission of ultrasonic vocalizations (Floody, Pfaff, \& Lewis, 1977). Gonadectomy and hormone replacement studies have demonstrated the dependence of some behavior patterns on a particular hormonal state of the animals, such as lordosis (Carter, Michael, \& Morris, 1973; Ciaccio, Lisk, \& Reuter, 1979) and agonistic behavior (Payne \& Swanson, 1971, 1972).

Although the literature indicates that many types of behavior are influenced by gonadal hormones, most of this research has examined only a single behavior pattern at a time; however, Richards (1966) and, more recently, Lisk and his colleagues (Lisk, Ciaccio, \& Catanzaro, 1983; Takahashi \& Lisk, 1983) studied several different behavior patterns concurrently. One would expect that an

We thank K. E. M. Bruce, B. N. Bunnell, and S. Schwartz for their helpful criticisms of earlier drafts of this manuscript. S. Meyer prepared the figures. Please direct all correspondence to: K. J. Forand, Department of Psychology, SUNY at New Paltz, New Paltz, NY 12561. increase in one type of activity would be accompanied by a decrease in at least one other type of activity. These changes should be reflected in systematic fluctuations in female time budgets across the estrous cycle. An estimate of the daily time budget has been given for male hamsters (Lerwill, 1974), but a detailed description of a daily time budget for females, with respect to the estrous cycle, is not available.

Several studies indicate the importance of social factors in determining the behavior of female hamsters. Floody et al. (1977) reported that females emitted lowlevel ultrasonic vocalizations at all stages of the estrous cycle, but introduction of a male or his odors significantly increased the amount of female vocalization. Social context has been shown to be important for the expression of lordosis as well (Beach, Stern, Carmichael, \& Ransom, 1976).

The purpose of the present study was to describe the time-budgeting practices of female hamsters with regard to their estrous cycles, and then to examine the effect of the presence of intact males and castrated males on that time budget.

\section{METHOD}

\begin{abstract}
Subjects
All animals used in this study were bred in our laboratory and were descendants of the Lakeview outbred hamster strain. Subjects were 6 female Syrian golden hamsters (Mesocricetus auratus) selected from a pool of 18 because they showed regular 4-day estrous cycles. Estrous cycles were monitored using the method described by Orsini (1961). Vaginal secretions were examined daily for discharges characteristic of each phase of the cycle. Day 1 of the cycle was characterized by the distinctive postestrous discharge, and Day 4 was the day of vaginal estrus. Subjects were housed in individual cages from the time of weaning
\end{abstract}


to approximately 100 days, when testing began. Purina Laboratory Chow and water were provided ad lib. The colony room was maintained on a 14-10 h light-dark cycle at a temperature of $24 \pm 2^{\circ} \mathrm{C}$.

Stimulus animals were 12 males chosen at random from a pool of 15 . Of the 12,6 were gonadectomized at approximately 80 days of age. The remaining 6 were assigned to the "intact" group and recieved no special treatment. Both groups of males were approximately 100 days old at the start of the study. They were reared in the same colony room as the females.

\section{Apparatus}

Testing was carried out adjacent to the colony in a room that was maintained at the same temperature and light cycle as the colony room, except that four $25-\mathrm{W}$ red bulbs were illuminated at all times to facilitate observations conducted during the dark phase. During testing, each subject was housed in a $40 \times 30 \times 20 \mathrm{~cm}$ clear plastic tub covered with a wire mesh roof. The floor of each tub was covered with $1-2 \mathrm{~cm}$ of Sani-cel bedding and cotton pellets were provided as nest material.

An enclosed wire mesh tube approximately $15 \times 7 \times 7 \mathrm{~cm}$ was placed in each tub. During the male-present conditions of the study, males were housed in these tubes. Each male had his own food and water supply.

\section{Procedure}

Test conditions. Data were collected for three conditions: female alone (FA), intact male present (IM), and castrated male present (CM). The FA condition was presented first to determine a baseline time budget with regard to the estrous cycle. The IM condition was presented second, followed by the CM condition. Observations began on the fifth day in each condition to provide females with a 4-day period (one complete estrous cycle) during which they might adjust to the new stimulus situation.

Method of observation. Data were collected using the instantaneous scan technique described by Altmann (1974). A cassette tape player delivered a low-intensity click at 30 -sec intervals. At the sound of the click, the behavior of each female was recorded as one of 20 patterns defined below.

Schedule of observation. A "composite" estrous cycle was formed by sampling and combining data from two consecutive cycles. Data were collected during four periods of $24 \mathrm{~h}$ each. Midpoints of the periods were lights-on, lights-off, and midpoints of the light and dark phases. Two periods were sampled on each day of one cycle, and the remaining periods were sampled on each day of the next cycle. The combined data provided a total of $8 \mathrm{~h}$ of observation for each day of the composite cycle.

Behavior categories. Previous work in this laboratory demonstrated that the behavior of female hamtsers can be accurately described with 20 categories of behavior defined by the motor acts or postures involved. For purposes of data analysis, these 20 behavioral categories were divided among six classes on the basis of their apparent or suggested function.

Maintenance consisted of patterns that served to support basic physiological functions. This class consisted of eating, drinking, gnawing, grooming, resting, and sleeping. Locomotion was bodily movement resulting in change of physical location in the environment: running or walking, climbing, nest building, and digging. Exploration consisted of patterns that served to maintain surveillance of the environment, including rearing, attentive posture, and sniffing. Hoarding, any foodrelated activity other than eating, included food carrying and food manipulation. Sex consisted of patterns on the part of the female which normally directly facilitate copulation: vaginal marking, prelordosis, and lordosis. Comfort behavior was a class consisting of patterns which interrupted bouts of sleep such as restless movement and stretching. A final notation " $X$ " was recorded for any scan on which the behavior of the subject did not resemble any of the patterns described above (such as urination) or for a scan on which the animal was not clearly visible.

Reliability of observation was established prior to the start of the study. Kappa, a statistic of reliability which corrects for chance agreements, was found to be .91 .

\section{Analyses}

The primary analysis was a two-way multivariate analysis of variance with both factors (day of cycle and social condition) treated as repeated measures. Significant main effects and simple main effects of significant interactions were assessed with post hoc Duncan's multiple range tests.

\section{RESULTS}

Females in FA spent $73 \%$ of their day engaged in maintenance activities. Sleep accounted for approximately $66 \%$ of all maintenance activity and $50 \%$ of the entire day. Females spent $15 \%$ of the day engaged in locomotor activity. Exploration accounted for $11 \%$ of the day. Food hoarding and comfort behavior each accounted for approximately $1 \%$ of the day. No sexual behavior was observed in FA. There were no statistically significant changes related to the estrous cycle.

Analyses showed that three behavior patterns, sniffing, digging, and restless movement, were influenced by the social condition variable. Two patterns, lordosis and food manipulation, were influenced by both social condition and day of the cycle.

The MANOVA for exploration activities showed a significant main effect of condition $[F(6,20)=4.14$, $p<$ $.01]$. This effect was due primarily to significant changes in sniffing $[\mathrm{F}(2,12)=9.13, \mathrm{p}<.01]$. Sniffing accounted for $8.6 \%$ of the day in IM and $7.5 \%$ in CM but only $3.7 \%$ in FA. Sniffing occupied significantly less time in FA than in both male-present conditions, but the IM and CM conditions did not differ significantly (Figure 1).

The MANOVA for locomotor activities showed a significant main effect of condition $[\mathrm{F}(8,18)=3.45$, $\mathrm{p}<$ $.05]$. This effect was due to significant changes in digging $[\mathrm{F}(2,12)=3.45, \mathrm{p}<.01]$. Digging accounted for $5.0 \%$ of daily activity in FA, but only $1.8 \%$ and $1.2 \%$ in IM and CM, respectively. Digging was significantly lower in FA than in both IM and CM, but again, IM and $\mathrm{CM}$ were not significantly different (Figure 1 ).

The MANOVA for comfort activites showed a significant main effect of condition $[\mathrm{F}(4,22)=3.17, \mathrm{p}<.05]$. This effect was due to significant changes in restless movement $[\mathrm{F}(2,12)=7.93, \mathrm{p}<.01]$. Restless movement ac-

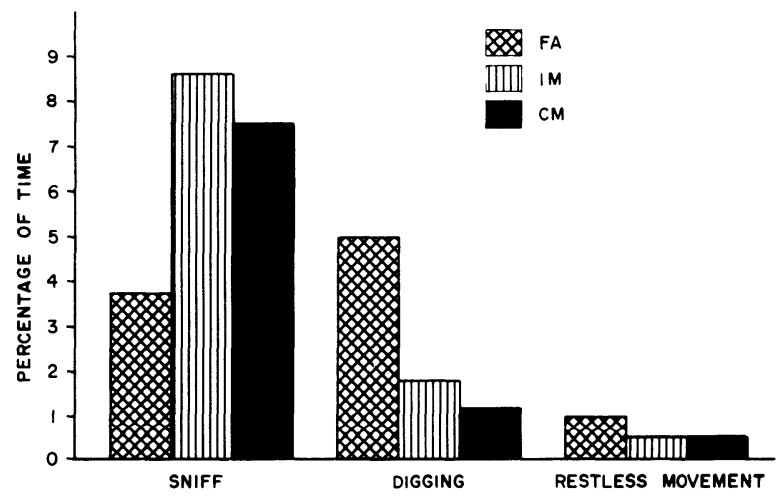

Figure 1. Effects of social condition on the percentage of time engaged in sniff, dig, and restless movement. FA = female alone, IM $=$ intact male, $\mathbf{C M}=$ castrated male. 


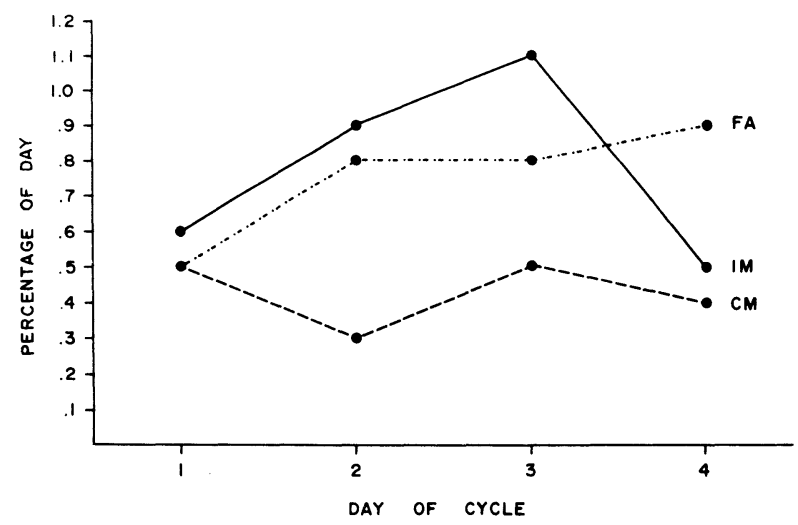

Figure 2. Percentage of time engaged in food manipulation across days of the estrous cycle for females in the FA, IM, and CM conditions. Day 4 is defined as the day of vaginal estrus.

counted for $1.0 \%$ of daily activity in FA but only $0.5 \%$ in both IM and CM. Again, differences between FA and male-present conditions were significant, but differences between IM and CM were not (Figure 1).

The MANOVA for sex activities could not be calculated because two components, vaginal marking and prelordosis, were not observed during the study. Therefore, the ANOVA on the remaining component, lordosis, was the only analysis available. There was a significant main effect of condition $[\mathrm{F}(2,12)=4.54, \mathrm{p}<.05]$, and a significant main effect of day of the cycle $[F(3,16)$ $=4.52, \mathrm{p}<.05]$. However, the condition $\times$ day interaction was also significant $[\mathrm{F}(6,20)=5.17, \mathrm{p}<.01]$. Lordosis occurred only in the presence of intact males and, in that condition, only on the day of estrus.

The MANOVA for hoarding behavior showed a significant condition $\times$ day interaction $[F(12,38)=2.74$, $\mathrm{p}<.01]$. This effect was due to changes in the component food manipulation $[F(6,20)=4.96, p<.01]$. There were no significant changes across days of the cycle in CM. There was a steady increase in the amount of time engaged in food manipulation in FA, but differences among the days were not significant. The interaction is due to a significant decrease in time engaged in food manipulation from Day 3 to Day 4 in the IM condition (Figure 2).

\section{DISCUSSION}

The baseline time budget for female hamsters in the female alone (FA) condition showed a strong emphasis on maintenance behavior. Locomotor and exploratory activity occupied the next largest percentages of time, respectively. The time budget observed for females is comparable to findings for male hamsters (Lerwill, 1974). Percentages of time engaged in sleeping, eating, digging, and nest building are nearly identical for males and females. Although females spent somewhat more time engaged in grooming and exploratory behavior than males, and males spent more time resting than females, it appears that the time budgets of males and females are quite similar.

Females spent more time engaged in locomotor behavior in the FA condition than in both male-present conditions (see Figure 1). Hamsters tend to be solitary-living animals (Hoffman, Robinson, \& Magelhaes,
1968). In their natural semidesert habitat, vital resources such as food, water, and shelter are widely scattered. Locomotor activity is required not only to find these resources but also to find potential mates. In the present study, food and water sources were abundant and readily available. Observed differences in locomotion probably reflect changes related to searching for a mate. In the male-present conditions, because the object of the search was provided in the immediate vicinity of the other resources, the need to wander was reduced.

That changes in locomotor activity were related to mate acquisition is further suggested by the observation that approximately $75 \%$ of the decrease in locomotor activity from FA to the male-present conditions was offset by an increase in exploratory sniffing. Although it cannot be directly demonstrated that the increased sniffing was directed at the males, compensatory changes in locomotion and exploration suggest a reciprocal relationship.

The restless movement component of comfort activity was observed more frequently in FA than in both male-present conditions. This was a surprising result. It was expected that the males might have provided additional stimuli to the environment which would have disturbed the sleep pattern and produced more restless movement in these conditions than that seen in the FA condition. At present, it is not clear what aspect of the social environment is responsible for this effect.

Lordosis was observed only in the presence of intact males. Beach et al. (1976) observed lordosis in females in the presence of both intact and castrated males. In their paradigm, however, females had free access to the males. It is likely that the caged castrated males in the present study did not provide the same stimulation (e.g., tactile) as the castrated males in their study. If this is so, then it seems reasonable to expect the responses of the females to be different. In addition, lordosis was observed only on the day of estrus when estrogen and progesterone were expected to reach their highest levels. This result is consistent with previous findings (Beach et al., 1976; Carter, et al., 1973). The interaction between social condition and day of the estrous cycle reaffirms that the expression of lordosis is dependent on both external social and internal hormonal cues.

The condition $x$ day interaction for the food manipulation component of hoarding was an interesting and unexpected finding. Although the significant decrease in time spent hoarding from Day 3 to Day 4 of the cycle would be predicted on the basis of Estep et al. (1978), the present data cannot be interpreted as a replication of their results due to procedural differences between the studies. They found that significant changes in food hoarding occurred across the estrous cycle when females were housed alone, but this was not found in our study. A significant effect was found only in the IM condition. This finding suggests that even nonsocial behavioral patterns such as food hoarding may be dependent upon the social environment. The change in food hoarding across days in the IM condition represents a shift from maintenance activities to mate acquisition for females on the day of estrus. This interpretation has been suggested previously by Estep et al. (1978).

In summary, the results of this study suggest three conclusions. First, the time budget of female hamsters housed alone appears very simialr to the time budget reported for male hamsters housed alone. Second, social context is an important determinant of behavior, even in basically solitary-living animals such as hamsters. This is evidenced by significant changes across social conditions in four of the six classes of behavior studied in this experiment. Third, expression of some behavior patterns, but not others, is dependent on both hormonal states of the animals and the social context in which they live. It is not surprising to find that social behavior patterns change as females shift their emphasis from bodily maintenance to mate acquisition during the estrous cycle. However, it is interesting to find that nonsocial patterns can be similarly influenced by this interaction.

\section{REFERENCES}

Altmann, J. (1974). Observational study of behavior: Sampling methods. Behaviour, 49, 227-267.

Beach, F. A., Stern, B., Carmichael, M., \& Ransom, E. (1976). Comparisons of sexual receptivity and proceptivity in female hamsters. Behavioral Biology, 18, 473-478. 
Beatty, W. W. (1979). Gonadal hormones and sex differences in nonreproductive behaviors in rodents: Organizational and activational influences. Hormones \& Behavior, 12, 112-163.

Carter, C. S., Michael, S. J., \& Morris, A. H. (1973). Hormonal induction of female sexual behavior in male and female hamsters. Hormones \& Behavior, 4, 129-141.

Ciaccio, L. A., Lisk, R. D., \& Reuter, L. A. (1979). Prelordosis behavior in the hamster: A hormonally modulated transition from aggression to sexual receptivity. Journal of Comparative \& Physiological Psychology, 93, 771-780.

Estep, D. Q., Lanier, D. L., \& Dewsbury, D. A. (1978). Variation of food hoarding with the estrous cycle of Syrian golden hamsters (Mesocricetus auratus). Hormones \& Behavior, 11, 259-263.

Floody, O. R., PfafF, D. W., \& Lewis, C. D. (1977). Communication among hamsters by high-frequency acoustic signals: II. Determinants of calling by females and males. Journal of Comparative \& Physiological Psychology, 91, 807-819.

Hoffman, R. A., Robinson, P. F., \& Magelhaes, H. (Eds.) (1968). The golden hamster: Its biology and use in medical research. Ames, Iowa: Iowa University Press.

LERWILL, C. J. (1974). Activity rhythms of golden hamsters (Mesocricetus auratus) and Mongolian gerbils (Meriones unguiculatus) by direct observation. Journal of Zoology, 174, 520-523.

LESHNER, A. I. (1979). An introduction to behavioral endocrinology. New York: Oxford University Press.

Lisk, R. D., Ciaccio, L. A., \& Catanzaro, C. (1983). Mating behaviour of the golden hamster under semi-natural conditions. Animal Behaviour, 31, 659-666.
Johnston, R. E. (1977). Causation of two scent-marking behavior patterns in female hamsters. Animal Behaviour, 25, 317-327.

Morin, L. P. (1978). Rhythmicity of hamster gnawing: Ease of measurement and similarity to wheel running. Physiology \& Behavior, 21, 317-320.

Morin, L. P., \& Fleming, A. S. (1978). Variation of food intake and body weight with estrous cycle, ovariectomy and estradiol benzoate treatment in hamsters (Mesocricetus auratus). Journal of Comparative \& Physiological Psychology, 92, 1-6.

ORSINI, M. W. (1961). The external vaginal phenomena characterizing the stages of the estrous cycle, pregnancy, pseudopregnancy, lactation and the anestrous hamster (Mesocricetus auratus Waterhouse). Proceedings of the Animal Care Panel, 11, 193-206.

Payne, A. P., \& Swanson, H. H. (1971). Hormonal control of aggressive dominance in the female hamster. Physiology \& Behavior, 6, 355-357.

Payne, A. P., \& Swanson, H. H. (1972). The effect of sex hormones on the aggressive behavior of the female golden hamster (Mesocricetus auratus Waterhouse). Animal Behaviour, 20, 782-787.

RicharDs, M. P. M. (1966). Activity measured by running wheels and observation during the oestrous cycle, pregnancy and pseudopregnancy in the golden hamster. Animal Behaviour, 14, 450-458.

TAKAHASHI, L. K., \& LISK, R. D. (1983). Organization and expression of agonistic and socio-sexual behavior in golden hamsters over the estrous cycle and after ovariectomy. Physiology \& Behavior, 31, 477-482.

(Manuscript received for publication February 25, 1985.) 